\title{
ANALYSIS OF DEFORMATION PROCESSES OF MAGNESIUM ALLOY AT ELEVATED TEMPERATURES BY ORIENTATION MAPPING
}

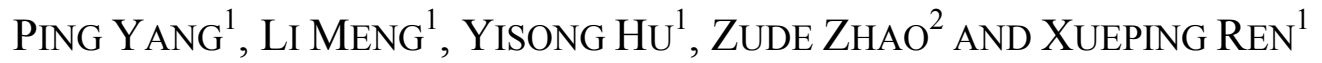 \\ ${ }^{1}$ School of Material Science and Engineering University of Science \& Technology Beijing, 100083, Beijing, \\ China; ${ }^{2}$ The $59^{\text {th }}$ Institute of Chongqing, 630000, Chongqing, China \\ e-mail: yangp@mater.ustb.edu.cn \\ (Accepted February 5, 2004)
}

\begin{abstract}
Orientation mapping based on electron back scattering diffraction technique was applied to reveal the distributions of disorientations and rotation axes of grains caused by plastic slip and twinning during channel die compression in magnesium alloy ZA31. In addition, the orientations of dynamically recrystallized grains and deformed grains were separated and compared with respect to their initial textures. The relationship of strain and $\left\{\begin{array}{ll}10 & \overline{1}\end{array}\right\}$ twin amount was determined quantitatively by referring to twin orientations. The reasons leading to the observed phenomena are analyzed and discussed.
\end{abstract}

Keywords: deformation, magnesium alloy, orientation mapping, recrystallization, texture.

\section{INTRODUCTION}

Orientation mapping based on electron back scattering diffraction is an effective technique developed in the 90' of last century (Dingley and Field, 1997; Adams et al., 1994) to reveal crystallographic structures and orientations. In addition to the morphological information abundant orientation data associated with sample position permits further analysis of disorientation and rotation axis distribution by orientation data processing. Therefore a comprehensive understanding of relevant deformation and recrystallization mechanisms in local scale can be achieved and a better material design and control of microstructures and properties are possible. Magnesium alloys are lightest structural materials and have been applied in automotive, aerospace and communication industries. During their processing plastic slip and twinning will proceed (Kelley and Hosford, 1968; Yoo, 1981). Moreover, dynamic recrystallization will prevail at elevated temperatures (Ion et al., 1982; Tan and Tan, 2003). These processes alter grains morphologies, their crystallographic orientations and orientation relationships in different ways, and therefore lead to distinct final properties. In this paper distributions of disorientations and rotation axes of grains caused by plastic slip and twinning were investigated in samples with different initial textures by means of orientation mapping. In addition orientation distribution of dynamically recrystallized grains with respect to their neighboring deformed grains was determined and compared with initial textures.

\section{MATERIALS AND METHODS}

Samples of dimension $15 \times 10 \times 6 \mathrm{~mm}$, called XZ, $\mathrm{ZY}, \mathrm{XY}$ and $\mathrm{RN}$, were prepared from a hot rolled magnesium alloy sheet (RN) and extruded bars AZ31 $(X Z, Z Y, X Y)$ with strong texture. The initial microstructures of samples $\mathrm{XZ}, \mathrm{ZY}$ and $\mathrm{XY}$ were very inhomogeneous with average grain size being $34 \mu \mathrm{m}$. Sample RN contained a uniform microstructure with a mean grain size of $25 \mu \mathrm{m}$. These samples were subject to plane strain compression (in channel die) at the temperature range of $97^{\circ} \mathrm{C} \sim 340^{\circ} \mathrm{C}$ and strain rate of $0.01 / \mathrm{s} . \mathrm{MoS}_{2}$ paste was applied to reduce friction between sample and channel die form. After compression samples were prepared using commercial AC-2 electrolyte and etched in picric-acid solution as described in detail elsewhere (Yang et al., 2003a). Xray diffraction was used to determine macro-texture. The EBSD system of Channel 4 mounted on SEM LEO-1450 was used to perform orientation mapping (Yang et al., 2001). To illustrate the distribution of crystal defect density (mainly referring to dislocation density) the map of Kikuchi band contrast, namely the Kikuchi band quality which is proportional to the dislocation density in grains and presented as an index ranged from gray degree of $0 \sim 255$, is often used. The higher this index, the less the crystal defect density in measured position. 


\section{RESULTS AND ANALYSES}

DISORIENTATION DISTRIBUTION

INFLUENCED BY $\left\{\begin{array}{ll}10 & \overline{1} 2\end{array}\right\}$ TWINNING

AND INITIAL TEXTURES

Fig. 1 shows the orientation mapping on three samples with different initial textures after a strain of $8 \%$ at $97^{\circ} \mathrm{C}$. The black color in Fig. 1a,e,i corresponds to basal orientation with $45^{\circ}$ scattering. The red and black thin lines refer to low angle grain boundaries of $5^{\circ}$ and $10^{\circ}$ respectively, while black thick line depicts high angle grain boundaries of over $15^{\circ}$. Although double prismatic slip (i.e., the concurrent activation of two $\{10 \overline{1} 0\}<11 \quad \overline{2} 0>$ slip systems $), \quad\{10 \overline{1} 2\}$ twinning and basal slip of $\{0001\}<11 \overline{2} 0>$ were determined to be the dominant deformation mechanisms in three samples respectively (Yang et al., 2003a), the influence of $\left\{\begin{array}{ll}10 & \overline{1}\end{array}\right\}$ twinning on disorientation distribution is more significant than plastic slip. This is evidenced by the distinct $86.3^{\circ}<1120>$ twin relationship in disorientation distribution and rotation axis distribution (Fig. $1 \mathrm{c}, \mathrm{d}, \mathrm{g}, \mathrm{h}, \mathrm{k}, \mathrm{l})$. By comparison it is clear that twinning played an overwhelming role in sample ZY (Fig. $1 \mathrm{e}, \mathrm{g}, \mathrm{h})$. Sometimes a peak at $30^{\circ}$ co-exists with the $\left\{\begin{array}{ll}10 & 12\end{array}\right\}$ twinning in disorientation distribution, which may be related with the second twinning of $\{10 \overline{1} 2\}$ twins.

\section{THE RELATIONSHIP BETWEEN $\{10 \overline{1} 2\}$ TWINS AND STRAIN AMOUNT}

For annealing twins abundant twin boundaries are co-related with twin amount. However, this is not always the case for deformation twins. In the latter case twin amount is proportional to the twin-oriented grains. Optic microstructure observation of deformed magnesium alloy AZ31 revealed (Klimanek and Poetzsch, 2002) that at increase of strain twin amount reached a maximum and then decreased to a constant value which was regarded to be due to secondary twinning.

Fig. 2 illustrates the evolution of twinning by orientation mapping on samples RN deformed at different strains at $97^{\circ} \mathrm{C}$. The initial orientations (yellow color) were located in the near of RD in pole figure and it is already known that during compression $\left\{\begin{array}{ll}10 & \overline{1} 2\end{array}\right\}$ twinning would lead to basal orientation (black color). Fig. $2 \mathrm{a}$ is an orientation mapping on a sample deformed at $0.5 \%$. The pole figure shows that only some grains rotated from initial orientations at $\mathrm{RD}$ to the basal orientation near ND. The strong peak at $85 \sim 90^{\circ}$ in Fig. 2c indicates the $\{10 \overline{1} 2\}$ twinning $\left(86.3^{\circ}<11 \overline{2} 0>\right.$ ). At strain of $1.9 \%$ (Fig. 2d) many coherent twin boundaries and a stronger basal texture were detected. Again the peak at $85 \sim 90^{\circ}$ is prevailing (Fig. 2f). When strain was increased to $9.3 \%$ most grains were rotated to basal orientation by twinning (black color). The initial texture was vanished, twin boundaries were reduced and therefore the peak at $85 \sim 90^{\circ}$ was not as apparent as before (Fig. 2i). Note that the subgrain boundaries (blue lines in Fig. 2g) inside grains evident the concurrent plastic slip. From orientation mapping, it is known that the strongest twin orientation relationship corresponded to the abundant coherent twin boundaries, whereas twin amount was co-related with the intensity of basal texture or the area fraction of basal oriented grains. 


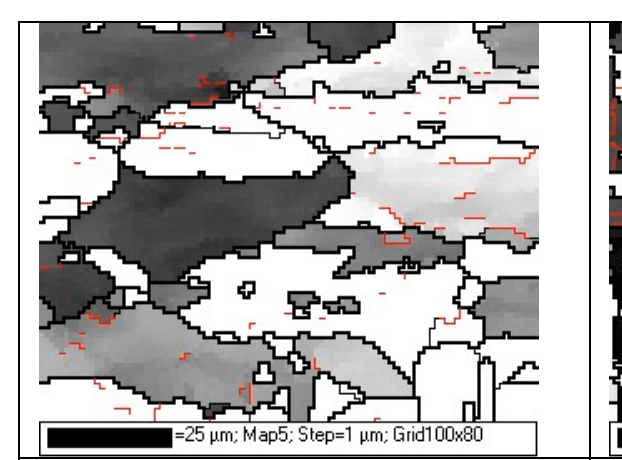

a) orientation mapping, $\mathrm{XZ}$

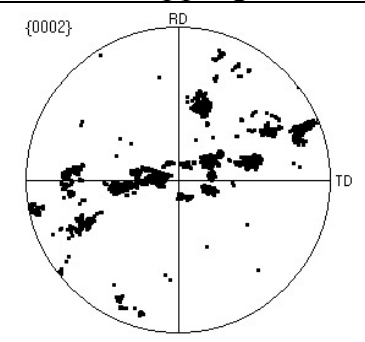

b) pole figure, $X Z$

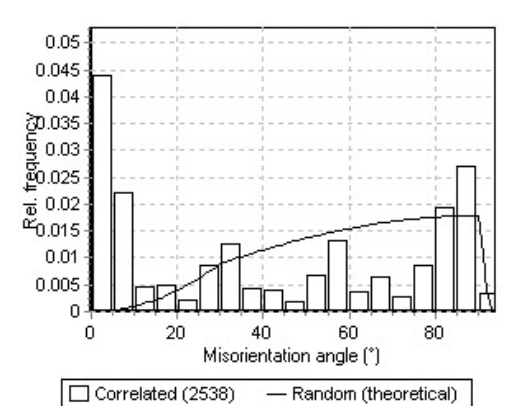

c) disorientation distribution, $\mathrm{XZ}$
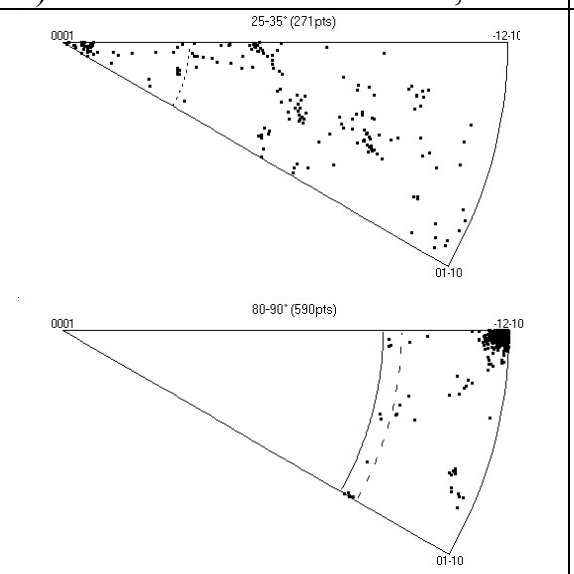

d) rotation axis distribution, $\mathrm{XZ}$

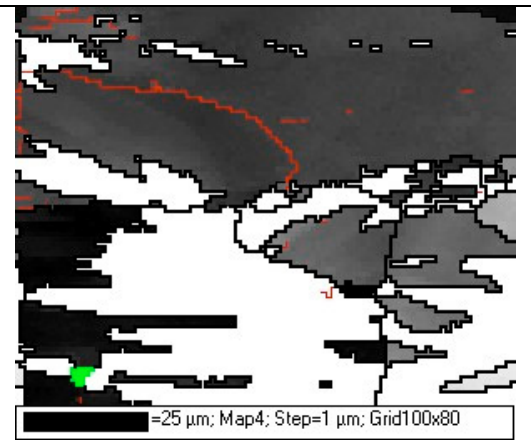

e) orientation mapping, ZY

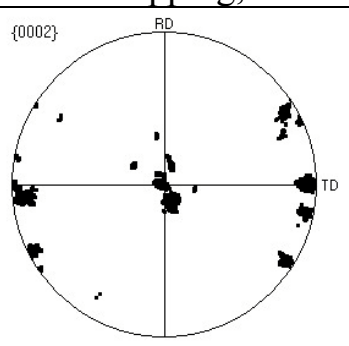

f) pole figure, $Z Y$

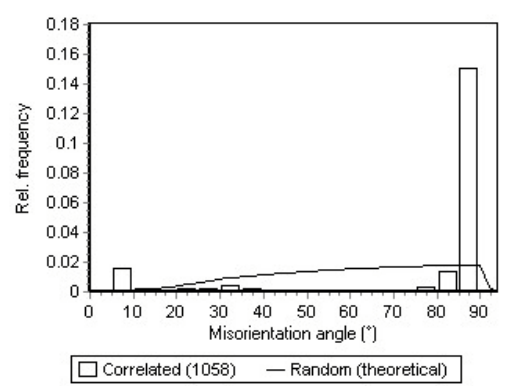

$\square$ Correlated (1058) -Random (theoretical)

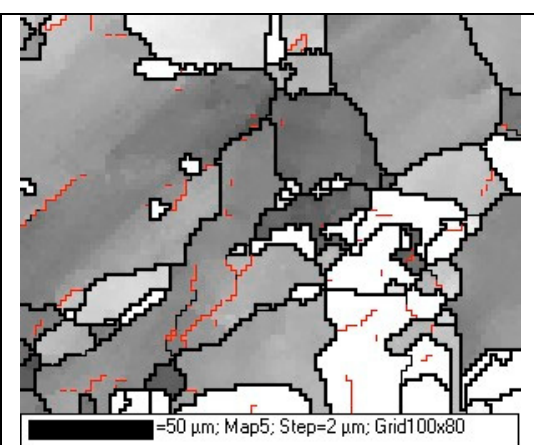

i) orientation mapping, $\mathrm{XY}$

g) disorientation distribution, ZY

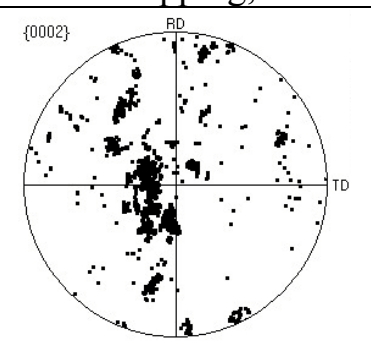

j) pole figure, $X Y$

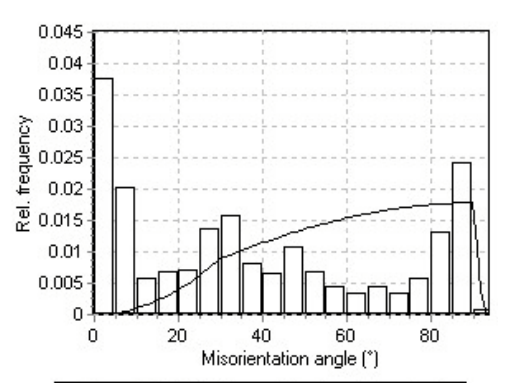

$\square$ Correlated (2100) -Random (theoretical)

k)disorientation distribution, $\mathrm{XY}$
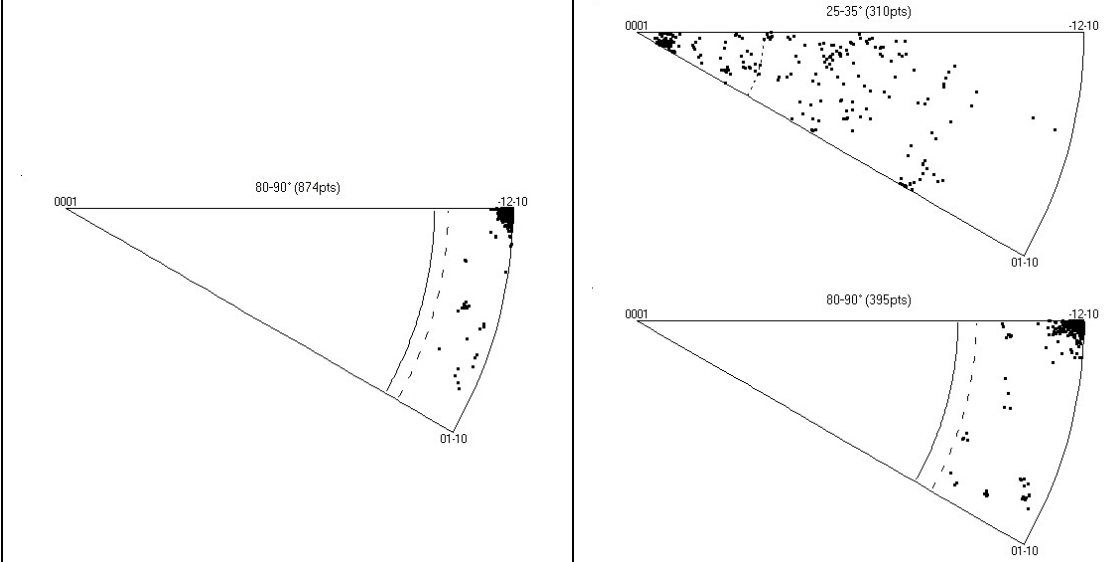

h) rotation axis distribution, ZY

1) rotation axis distribution, $X Y$

Fig. 1. Distribution of disorientation influenced by twinning and initial textures, samples were deformed by $\varepsilon=8 \%$ at $97^{\circ} \mathrm{C}$. 


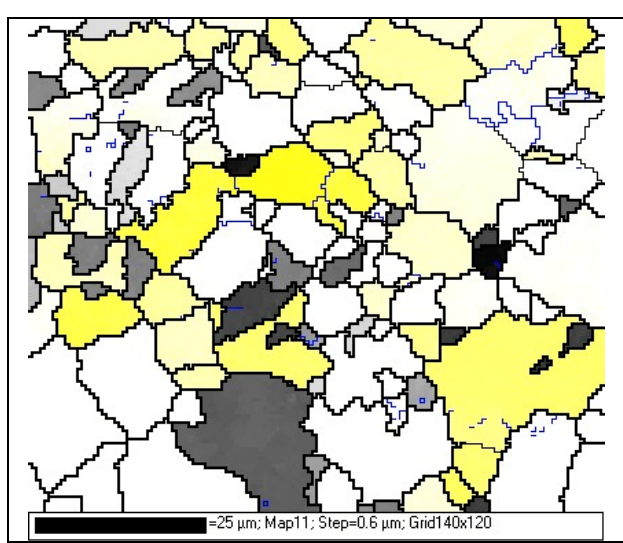

a) orientation mapping, $0.5 \%$

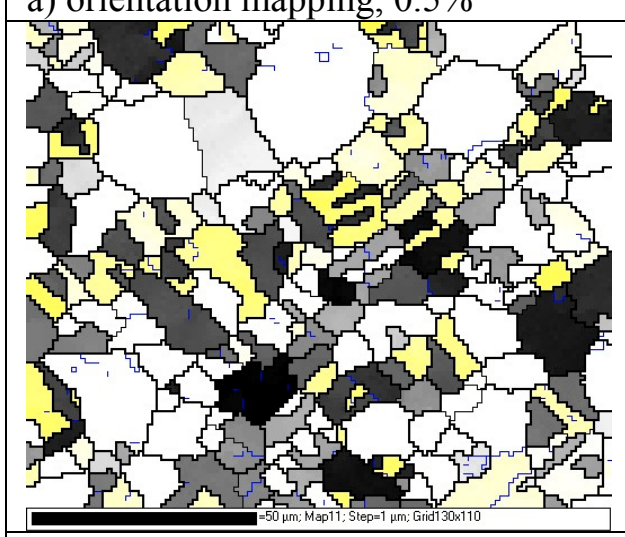

d) orientation mapping, $1.9 \%$

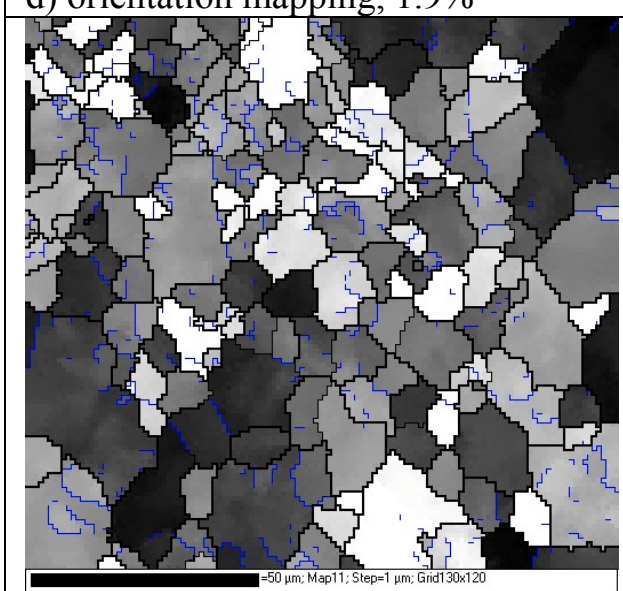

g) orientation mapping, $9.3 \%$

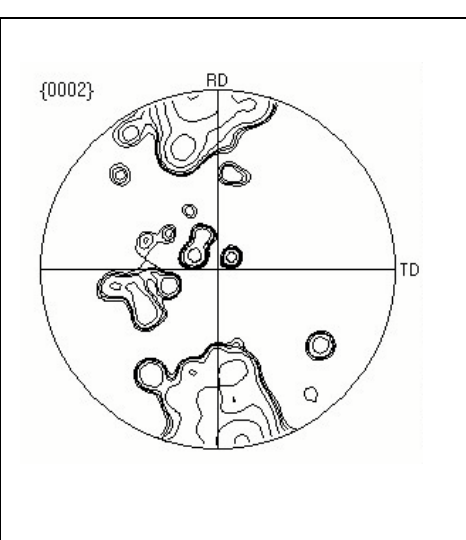

b) pole figure of (a)

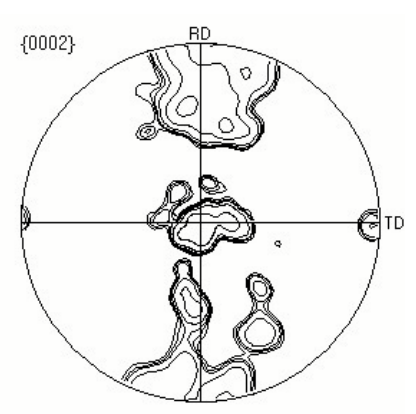

e) pole figure of $(d)$

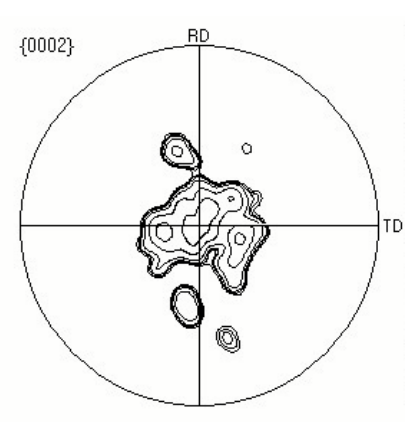

c) disorientation distribution of (a)

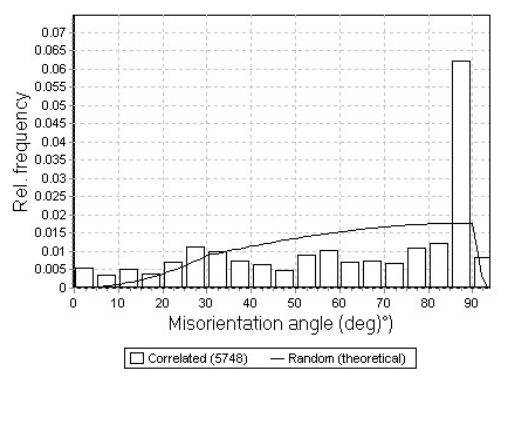

f) disorientation distribution of $(\mathrm{d})$

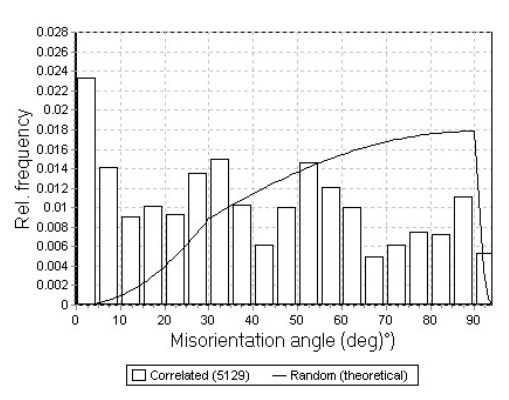

i) disorientation distribution of $(\mathrm{g})$

Fig. 2. Evolution of microstructures during straining revealed by orientation mapping data in sample RN deformed at $97^{\circ} \mathrm{C}$. The yellow color stands for initial orientations in the near of $R D$ position of pole figure, while black color refers to basal orientation in the near of ND position of pole figure.

Fig. 3 shows the relationship of strain and the area fraction of basal oriented grains, i.e., twin amount. As the $\mathrm{C}$-axes of most grains in initial samples were parallel to $\mathrm{RD}$ and the $\{10 \overline{1} 2\}$ twinning lead to an abrupt orientation change of $86.3^{\circ}$, only basal texture of $<0001>\| \mathrm{ND}$ was resulted during plane strain compression and the area fraction of basal oriented grains should be accounted to the twin amount. In consideration of the less statistic data of one orientation map, the results of 3 4 orientation maps for each strain were summarized. A scatter of $45^{\circ}$ with respect to ideal basal orientation is permitted because two variants of twinning may occur which were $60^{\circ}$ apart to each other. It is seen from Fig. 3 that a monotonic increase of twin amount with strain exists, rather than a maximum peak (Klimanek and Poetzsch, 2002). 


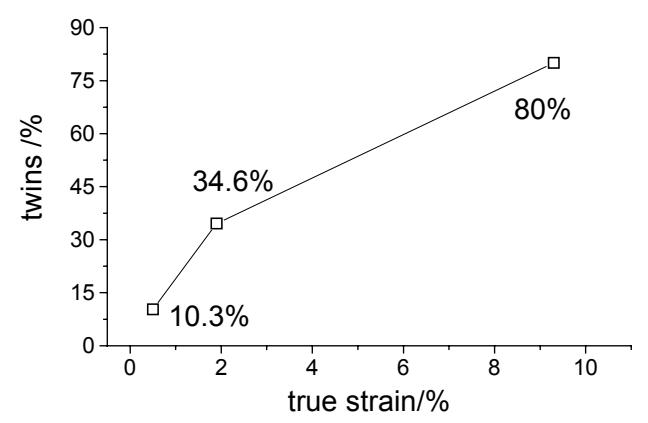

Fig. 3. Relation of strain amount and area fraction of basal oriented grains formed by twinning.

\section{ROTATION AXIS DISTRIBUTION OF LOW ANGLE GRAIN BOUNDARIES}

The distribution of rotation axis of low angle grain boundaries should reflect the activated plastic slip mechanisms to some extent and the prismatic slip dominated in sample $\mathrm{XZ}$ should produce a different distribution of rotation axes with respect to the basal slip prevailing in sample XY. Whether this difference in the distribution of rotation axis can be detected is still not aware. For deformation-induced low angle grain boundaries two types can be distinguished (KuhlmannWilsdorf and Hansen, 1991). One is incidental dislocation boundary (IDB) and the other is geometrical necessary boundary (GNB). The former refers to the dislocation cells with smaller disorientation, whereas the latter corresponds to dense dislocation walls or microbands with larger disorientation. The rotation axis distribution of IDBs is regarded to be random and that of GNBs is presumed to be preferred, usually, around TD. So far the information about this is very limited. Fig. 4 shows some examples in different samples. Fig. 4a gives the distribution of rotation axes in sample XZ and its morphology is shown in Fig. 1a. A weak preference at $<11 \quad \overline{2} 0>/<1 \quad \overline{1} 00>$ can be seen, which is contrary to the expected $<0001>$ preference by prismatic slip. A weak preference of $<11 \quad \overline{2} 0><<1 \quad \overline{1} 00>$ was also detected in sample XY (Fig. 4b) as expected by dominant basal slip. Its morphology is shown in Fig. 1i. Fig. 4c,d and Fig. 4e,f present two cases of low angle distributions within a coarse grain in samples ZY. The Fig. 4d exhibits the rotation axis distribution within a basal-oriented coarse grain deformed by $25 \%$ at $97^{\circ} \mathrm{C}$. Shear bands were developed inside this grain together with some subgrain boundaries (red lines). Shear bands are parallel to $\left\{\begin{array}{ll}10 & \overline{1} 1\end{array}\right\}$ planes and not to basal planes. A nearly random distribution is observed. Fig. 4e reveals the distribution of rotation axes within a $\{10 \overline{1} 2\}$-twined and basal-oriented coarse grain deformed by $60 \%$ at $266^{\circ} \mathrm{C}$. Red thin lines are subgrain boundaries. In this case a preference toward $<1$ 100>, i.e., the TD of sample, can be seen.

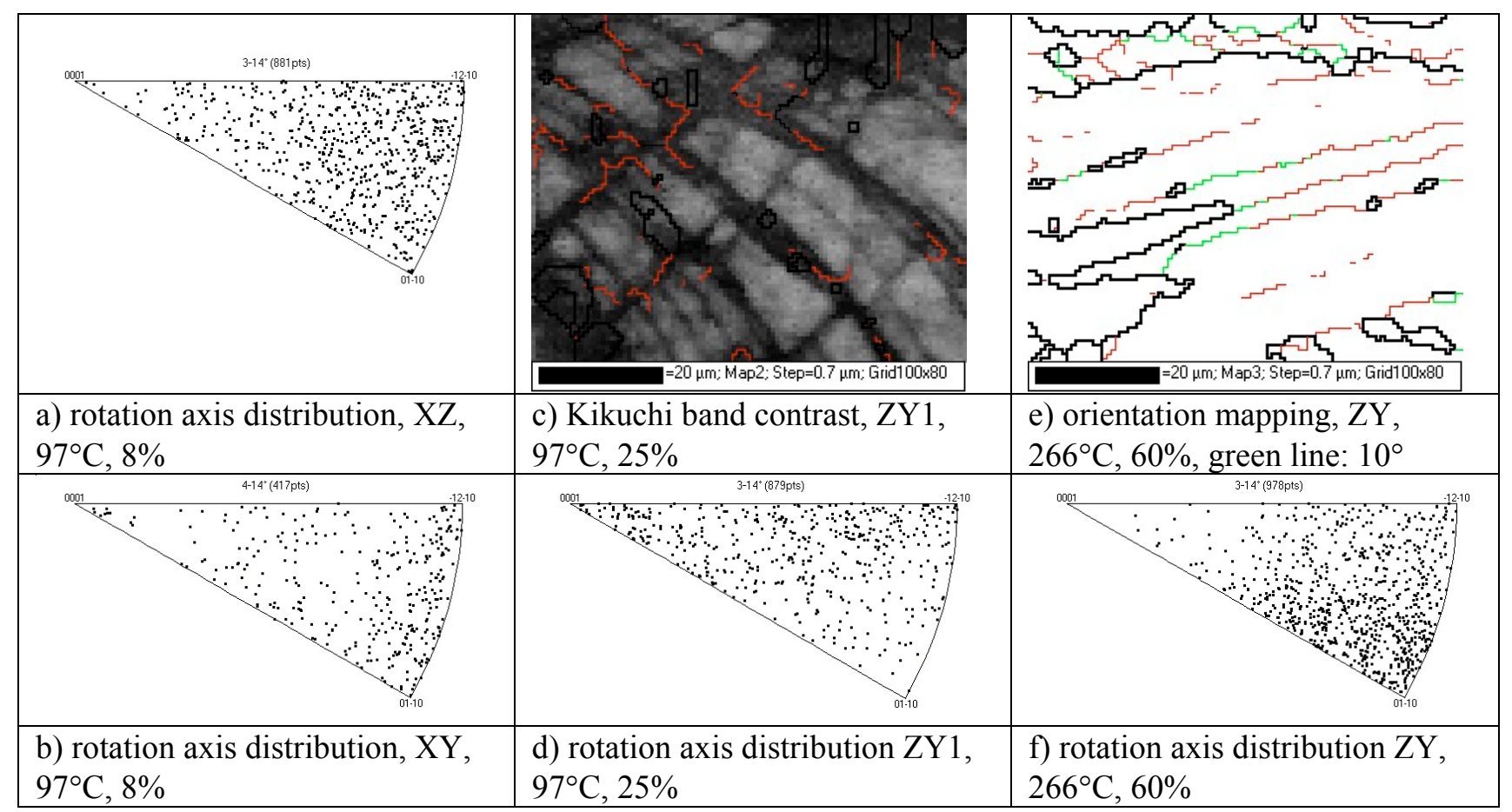

Fig. 4. Rotation axis distribution of low angle grain boundaries in different samples. 
Despite of the occurrence of superplasticity at high deformation temperature plastic slip still plays an important role. Fig. 5 shows the distribution of rotation axes in sample $\mathrm{XZ}$ at early stage of dynamic recrystallization at $266^{\circ} \mathrm{C}$. Red lines in Fig. 5a depict the low angle grain boundaries. The pole figure in Fig. 5b reveals many prism-oriented grains, i.e., the massive activity of prismatic slips. Preferences at $<0001>$ as well as at $<11 \overline{2} 0>/<1 \quad \overline{1} 00>$ can be seen (Fig. 5c).

Fig. 6 shows an orientation mapping on sample
$\mathrm{XY}$ deformed by true strain of $100 \%$ (equal to a compression by $63 \%$ in thickness reduction) at $266^{\circ} \mathrm{C}$. Although dynamic recrystallization was initiated at the strain of $17 \%$, it was not complete even at strain of $100 \%$. Due to strong basal texture developed at this period large angle grain boundaries over $40^{\circ}$ were rather less (Fig. 6c). A weak preference at $<0001>$ for both low and high angle grain boundaries can be seen (Fig. 6d,e). It is not clear whether this was related with strong basal texture or corresponded to prismatic/pyramid slips.

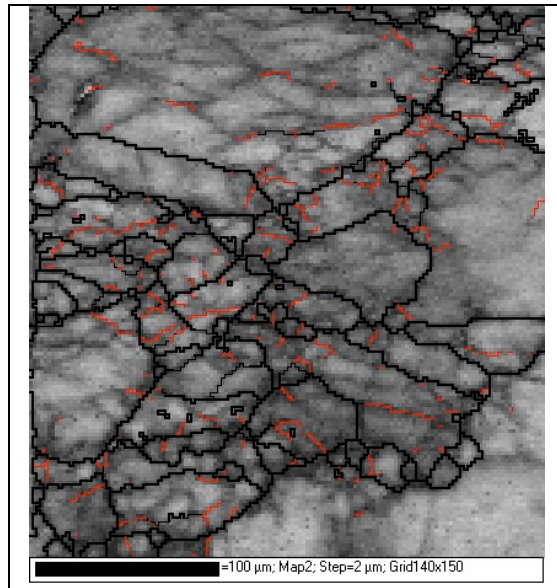

a) Kikuchi band contrast

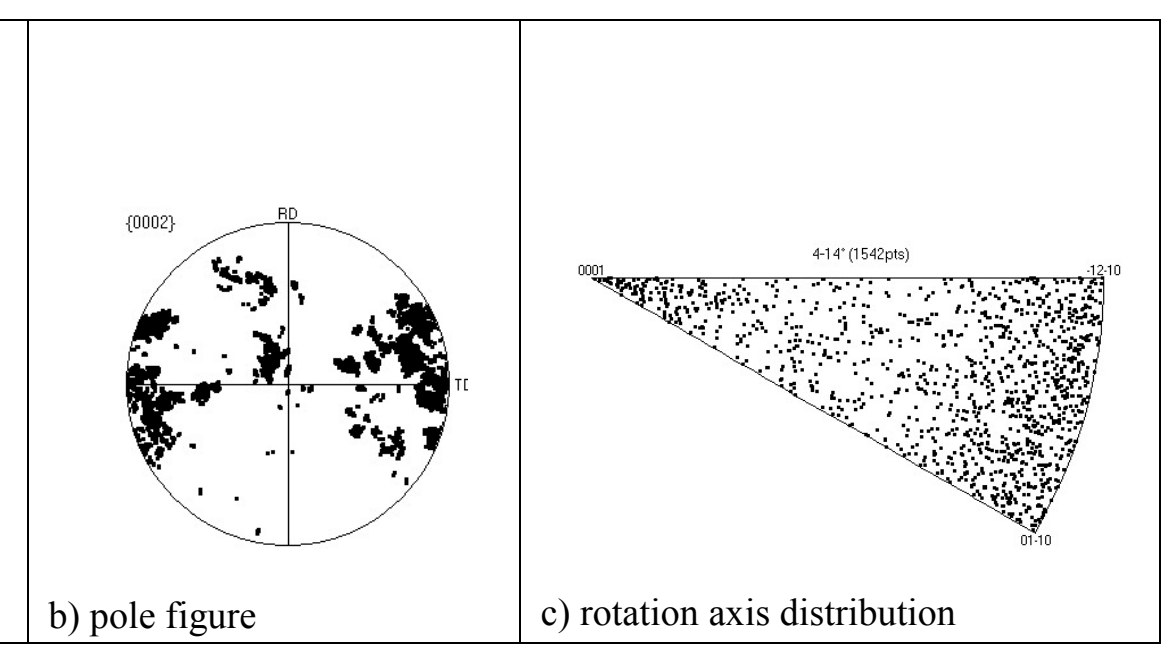

b) pole figure c) rotation axis distribution

Fig. 5. Rotation axis distribution at early stage of dynamic recrystallization in sample XZ deformed by strain $25 \%$ at $266^{\circ} \mathrm{C}$.

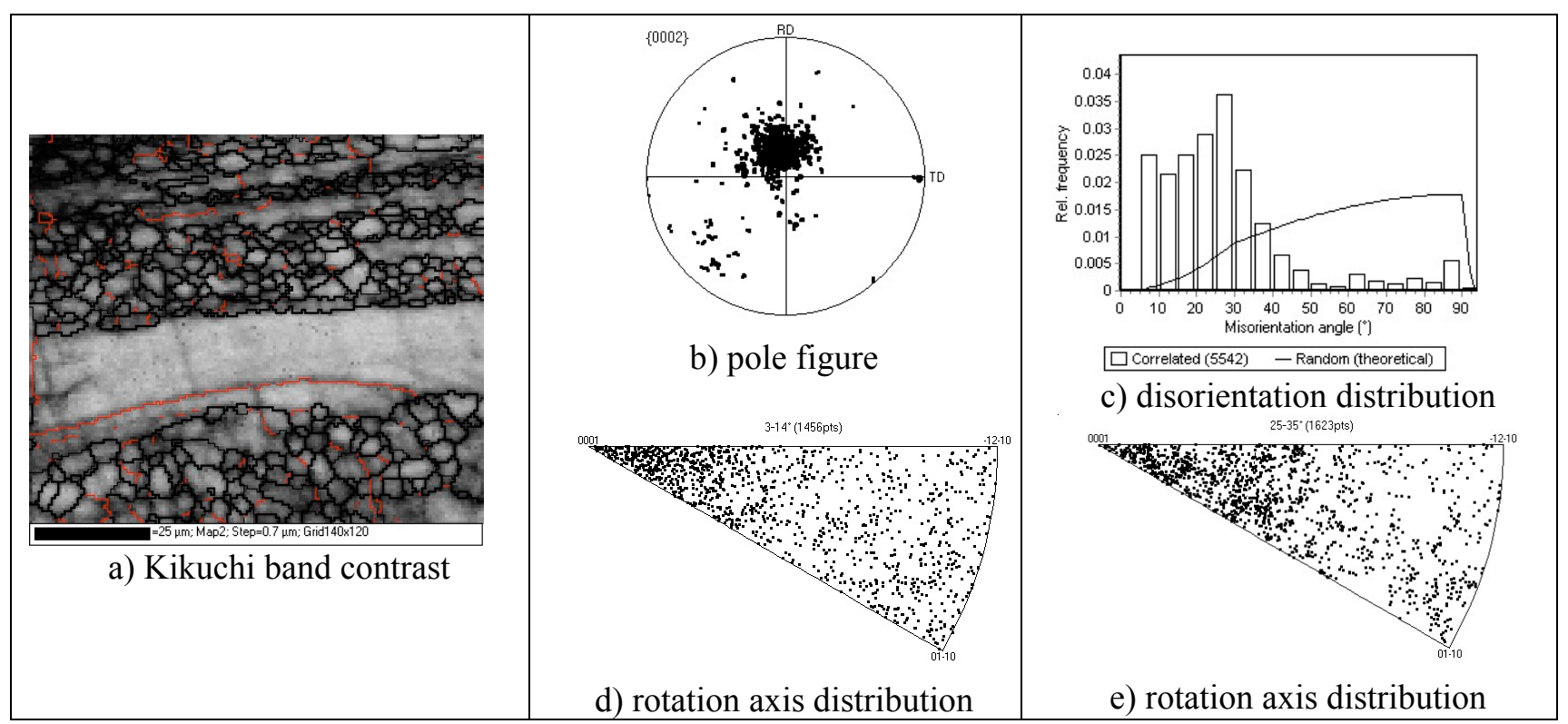

Fig. 6. Rotation axis distribution of low angle grain boundaries during dynamic recrystallization in sample $X Y$ deformed by true strain $100 \%$ at $266^{\circ} \mathrm{C}$. 
Large number of orientation mappings indicates that no totally random distribution of rotation axes was detected, nor was a clear relationship between initial textures and rotation axis distribution found. For instance, no clear preference of $<0001>$ was found in sample $\mathrm{XZ}$ with dominating double prismatic slips. In sample XY with prevailing basal slip no preference at $<$ uvt $0>$ was always found. A relative high noise (zero solution) during orientation mapping, which was due to the difficulty in sample preparation, may account to one reason. Further data collection is needed.

\section{ORIENTATIONS OF DYNAMICALLY RECRYSTALLIZED GRAINS}

Previous works indicated that initial textures influenced the volume fraction of recrystallized grains, the texture evolution and texture intensities (Yang et al., 2003b). Fig. 7 shows the textures of three samples deformed at $340^{\circ} \mathrm{C}$ by strain of $60 \%$ indicating the influence of initial textures. However, the macro-textures determined by X-ray diffraction can not provide clearly the information of recrystallized grains. Therefore, orientation mapping was utilized to reveal orientations of new grains.

Fig. 8 shows the orientations of recrystallized grains with respect to deformed matrix by separating recrystallized regions from those of deformed grains in three types of samples. It is seen that in general the deformed and recrystallized grains have similar orientations demonstrating the feature of dynamic continuous recrystallization (Tan and Tan, 2003). However, since their initial textures were different, the orientation spectrum of recrystallized grains was also distinct, either strongly scattered as in samples $\mathrm{XZ}$ and $\mathrm{ZY}$ or concentrated at basal orientation position as in sample $\mathrm{XY}$.

\section{CONCLUSIONS AND SUMMARIES}

1. In samples with different initial textures $\left\{\begin{array}{ll}10 & \overline{1}\end{array} 2\right\}$ twinning changed significantly the disorientation distribution producing the strong $86.3^{\circ}<11 \quad \overline{2} 0>$ relationship.

2. It is the volume fraction of basal-oriented grains, not the twin relationship, that co-relates with twin amount. A quantitative monotonic increase relation between strain and twin amount is determined by orientation mapping in sample $\mathrm{RN}$.

3. The distributions of rotation axes of low angle grain boundaries in different samples are not totally random, rather weak preference in $<0001>$ or $<1 \overline{1} 00>/<11 \overline{2} 0>$ can be found. Even in dynamically recrystallized samples preference of rotation axis distribution may also exist. The underlined mechanisms should be further investigated.

4. During dynamic recrystallization the orientations of new grains are similar to their deformed matrix indicating the feature of dynamic continuous recrystallization. However, the initial texture exerts strong influence on the orientation spectrum of new grains.

\section{ACKNOWLEDGMENT}

Financial supports from the National Nature Science Foundation of China (No:50171009) and from the National 863 projects (No.2002AA305501) are gratefully acknowledged.

"The preliminary form of this paper was originally presented at the $\mathrm{XI}^{\text {th }}$ International Congress for Stereology-Beijing Conference, Beijing, China, 4-8 November 2003."

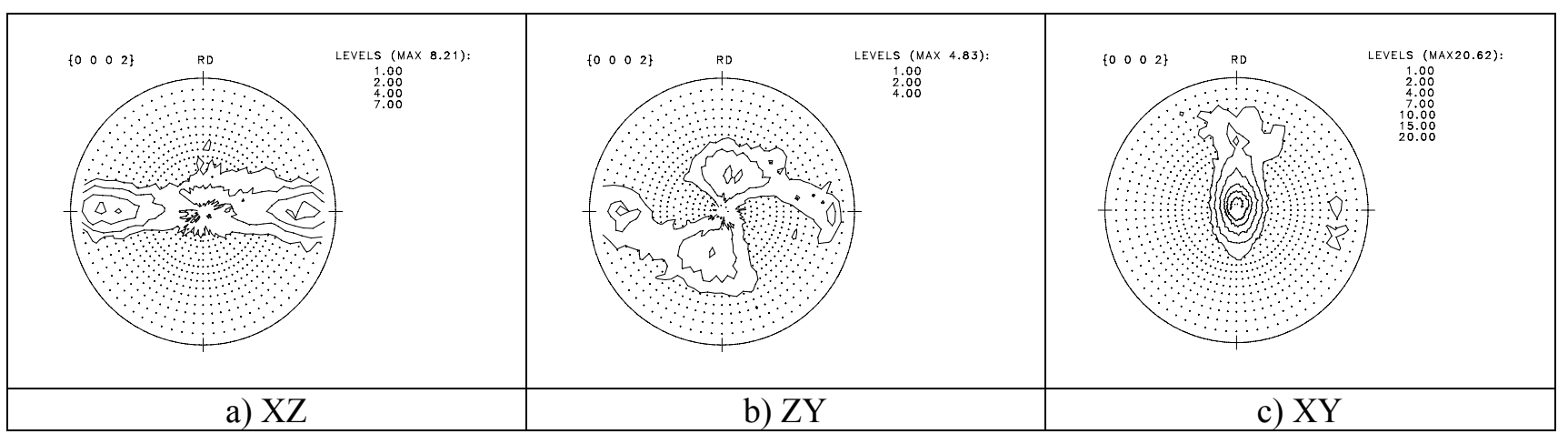

Fig. 7. Macro-textures determined by X-ray diffraction in three samples deformed by $\varepsilon=60 \%$ at $340{ }^{\circ} \mathrm{C}$. 


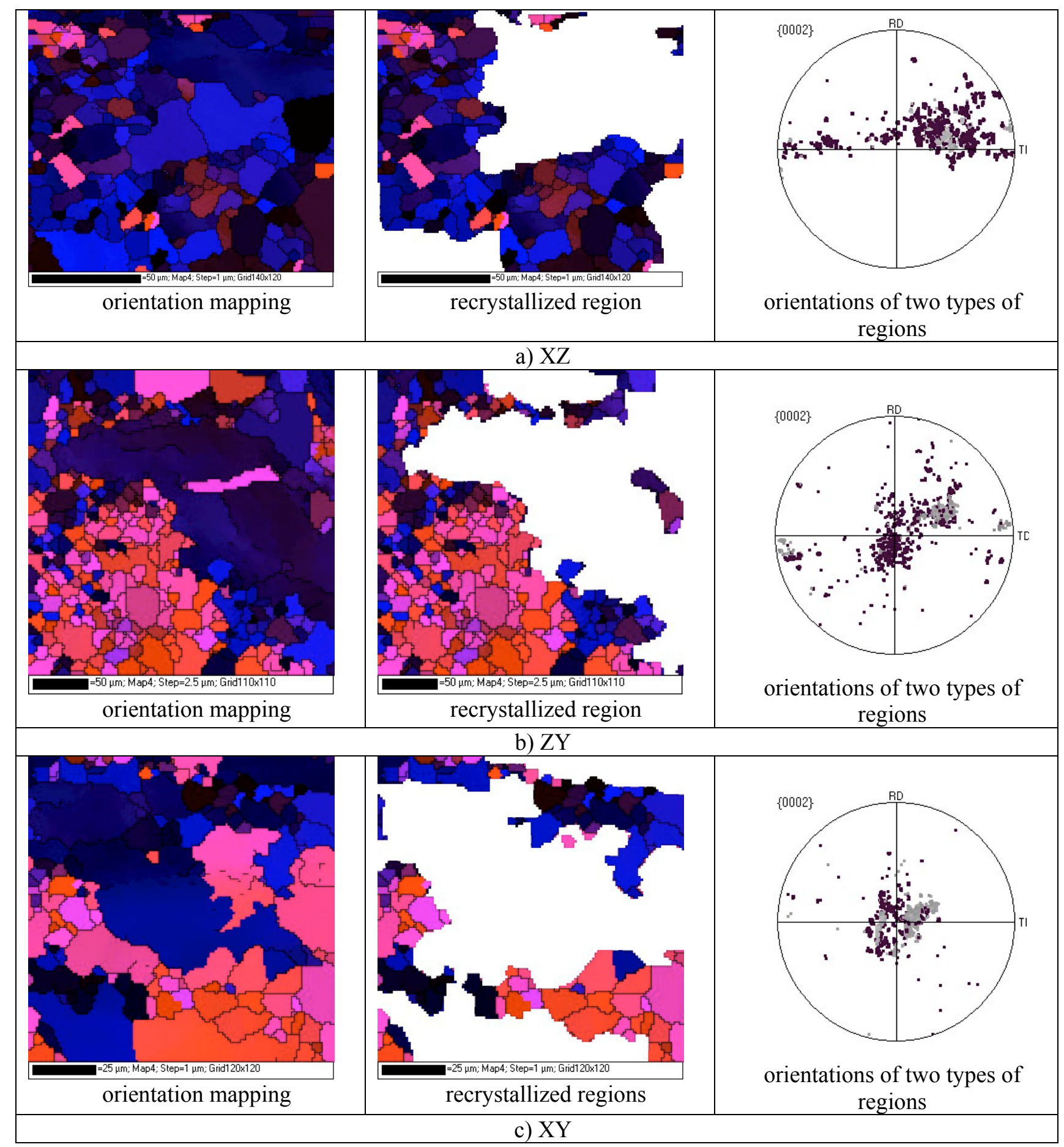

Fig. 8. Orientations of dynamically recrystallized grains with respect to deformed matrix from orientation mapping data, $340^{\circ} \mathrm{C}$, strain $60 \%$, different colors depict only different orientations. The dark color in pole figures represents the orientations of recrystallized grains, whereas the light color refers to orientations of deformed matrices. 


\section{REFERENCES}

Adams BL, Dingley DJ, Kunze K, Wright SI (1994). Orientation imaging microscopy: new possibilities for microstructural investigations using automated BKD analysis. Mater Sci Forum 157-162:31-42.

Dingley DJ, Field DP (1997). Electron backscatter diffraction and orientation imaging microscopy. Mater Sci \& Tech 13:69-78.

Ion SE, Humphreys FJ, White SH (1982). Dynamic recrystallisation and the development of microstructure during the high temperature deformation of magnesium. Acta metall 30:1909-19.

Kelley EW, Hosford WF, Jr (1968). Plane-strain compression of magnesium and magnesium alloy crystals. Trans AIME 242:5-13.

Klimanek P, Poetzsch A (2002). Microstructure evolution under compressive plastic deformation of magnesium at different temperatures and strain rates. Mater Sci \& Eng A324:145-50.
Kuhlmann-Wilsdorf D, Hansen H (1991). Geometrically necessary, incidental and subgrain boundaries. Scripta metall mater 25:1557-62.

Tan JC, Tan MJ (2003). Dynamic continuous recrystallization characteristics in two-stage deformation of Mg-3Al1Zn alloy sheet. Mater Sci \& Eng A339:124-32.

Yang P, Sun Z, Mao W (2001). Orientation mapping, an effective technique to analyze the microstructure, crystallographic structure and orientations of polycrystalline materials. Chinese Journal of Stereology and Image Analysis 6:50-4, 32 (in Chinese)

Yang P, Cui FE, Bian JH, Gottstein G (2003a). Relationships between deformation mechanisms and initial textures in polycrystalline magnesium alloys AZ31. Trans Nonferrous Met Soc China 13:280-4

Yang P, Cui FE, Ma SC, Gottstein G (2003b). Influence of initial textures on dynamic recrystallization and textures in AZ31 magnesium alloys. Trans Nonferrous Met Soc China 13:504-8

Yoo MH (1981). Slip, twinning, and fracture in hexagonal close-packed metals. Metall Trans 12A:409-18. 